\title{
A method to overcome wireless communication limits in decentralized active control applications
}

\author{
N. Debattisti ${ }^{a}$, M. L. Bacci ${ }^{a}$, S. Cinquemani ${ }^{a}$ \\ ${ }^{a}$ Politecnico di Milano, Dipartimento di Meccanica, Via La Masa 1, 20156 Milano, Italy.
}

\begin{abstract}
In this work the use of many stand-alone devices is considered for active vibration control. A stand-alone device represents a control unit which is able to independently perform the vibration control task, since it is embedded with sensors, an inertial actuator and a microcontroller, in which the control algorithm is implemented. The developed active controller is also embedded with a wireless module, in order to share information with other devices within the network. The proposed solution aims to improve the performance a decentralized control architecture and it is based on the optimal control theory. The Linear Quadratic Regulator works with the fullstate of the system, which is provided through a state estimation. A state recovery algorithm is then adopted to improve the quality of the estimation without placing a hefty burden on the wireless channel. Numerical analysis is made in order to study the advantages of this method. Finally, the proposed solution is validated with experimental results from a clamped-clamped beam.
\end{abstract}

Keywords: Vibration control, Inertial actuators, Control algorithms, Wireless sensors, Automation

\section{INTRODUCTION}

Among the different solutions adopted in structural vibrations control, active control systems can be a valid alternative to passive dampers, as they provide higher performance, especially for low-frequency applications. To date, the most used active control strategies are based on centralized architectures in which, as the size of the control system increases, logistical and implementation problems can occur, representing a limitation. ${ }^{1}$ In a totally decentralized control strategy, on the contrary, devices act as self-sufficient local controllers: ${ }^{2}$ since there is no share of information, the control action will depend only on local information, resulting in lower performance with respect to the centralized case. ${ }^{3,4}$ Decentralized static gain control is the simplest form of feedback control. If it is applied in a stable system where the sensors and actuators are colocated and dual, then stability is, in theory, guaranteed..$^{5,6}$

These architectures require a complex setup of all the components involved in the control task: many sensors and actuators have to be installed ${ }^{7,8}$ and wired to a real-time board, which manages the input signals coming from sensors and, through the implementation of the control algorithm, the output signals to provide to the actuators. To overcome this problem, a stand-alone device has been developed, which is fitted with all the necessary elements to work in an autonomous way: there are sensors for the data acquisition, a microcontroller in which the control algorithm is implemented and the actuator, driven by the board.

In this paper the possibility of exploiting a partial sharing of data between devices is investigated, in order to improve the overall quality of the vibration control of a totally decentralized architecture. To allow an easy placement of smart dampers and to avoid complex setup, wireless communication is used instead of wired connections. However, the use of this technology involves some complications that must necessarily be considered during the development phase: if the communication channel is saturated, in fact, phenomena such as data loss ${ }^{9,10}$ and transmission delay ${ }^{11}$ commonly occur, affecting the optimality and the stability of the control system. ${ }^{12,13}$ The proposed control strategy is based on the optimal control theory; ${ }^{14}$ the Linear Quadratic Regulator works with the full-state of the system, which is not available in similar applications. In order to provide the entire state vector, state estimation is adopted at each wireless sensor; identical Kalman filters, based on the same model of

Further author information: (Send correspondence to Simone Cinquemani) E-mail: simone.cinquemani@polimi.it Telephone: +39 (0)2 2399.8454 
the system, are implemented in all devices. The quality of the estimated state will limit the performance of the controlled system, since only one measure is available to correct the state evolution. For this reason, the wireless communication channel is exploited; the process which allows this improvement is called state recovery. ${ }^{15}$

The paper is structured as follows. In Section 2 the features of the stand-alone device are presented, while in Section 3 the mathematical model of the system is derived and the LQR and Kalman filter are defined. In Section 4 the state recovery algorithm is explained and the synthesis of the controller is made. In Section 5 the configuration of the system which has been used to perform the numerical analysis is presented and the corresponding results are shown. Finally, in Section 6 the experimental results obtained on a large vibrating system are shown, while conclusions are drawn in Section 7.

\section{THE STAND-ALONE DEVICE}

The developed device (shown in Fig. 1) is able to carry out operations of vibration control in an autonomous way. ${ }^{16,17}$ Two MEMS digital accelerometer are available: one is placed on the fixed frame of the device and the other on the suspended mass of the actuator. The first sensor measures the acceleration of the structure, which will be used to compute the control signal, while the second one measures the acceleration of the actuator, in order to get information about its dynamics during the control action.

These signals are acquired by the microprocessor, the main element of the electronic board in which different control algorithms can be implemented. ${ }^{18-20}$ The real-time control loop, triggered by the data-ready interrupt coming from the digital accelerometer placed on the fixed frame, runs at $1600 \mathrm{~Hz}$. The input signals are filtered in order to cancel out the static acceleration and other contributions which can lead to instability problems; the output of the control algorithm is the command signal: in order to drive the actuator, this signal must be converted from digital to analog, conditioned and amplified by the analog and power stages (see the Actuation stages in Fig. 2). The actuation system consists of a magnetodynamics inertial actuator, which can be modeled as a mass-spring-damper 1-DOF system excited by means of an electromagnetic force. ${ }^{21-23}$ The dynamics of this kind of actuators ${ }^{24}$ must necessarily be considered during the design phase of the control action; under their resonance frequency, inertial actuators generate a force with a small module and which is in counter-phase with respect to the vibration signal, leading to instability problems. ${ }^{25-28}$ The electronic board is also provided with a wireless module, which allows the communication with the other stand-alone devices (both transmission and reception). The wired communication with the computer is needed for the firmware loading and for the

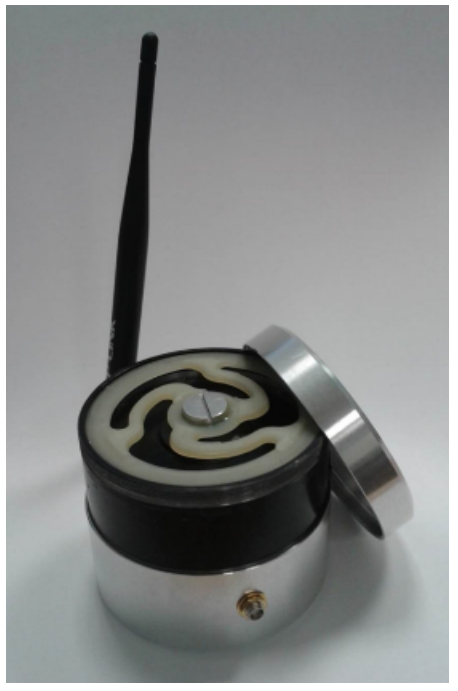

(a)

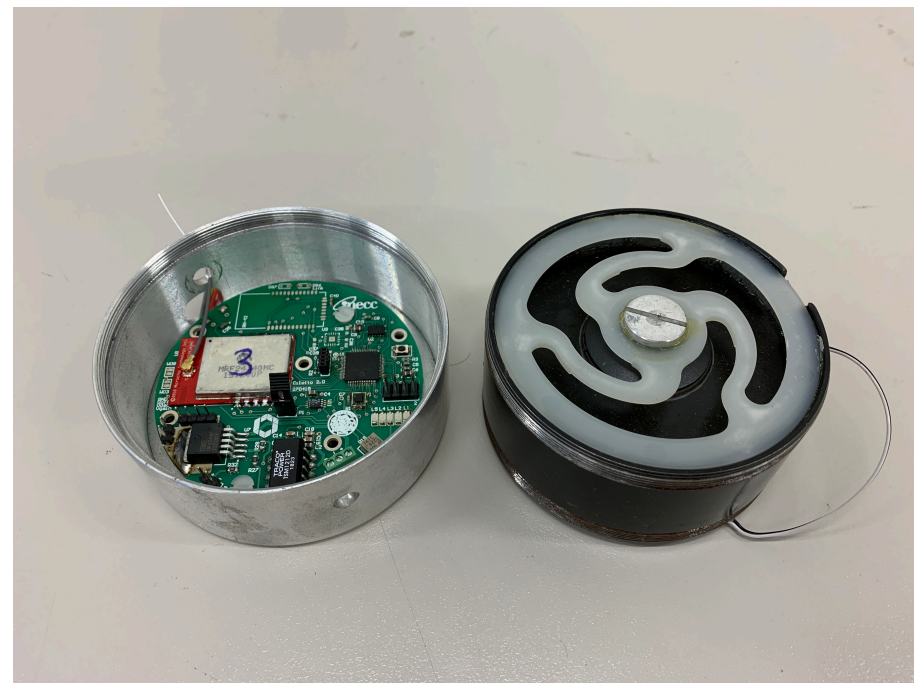

(b)

Figure 1: First prototype of the stand-alone device (a) and particular of the board (b) 


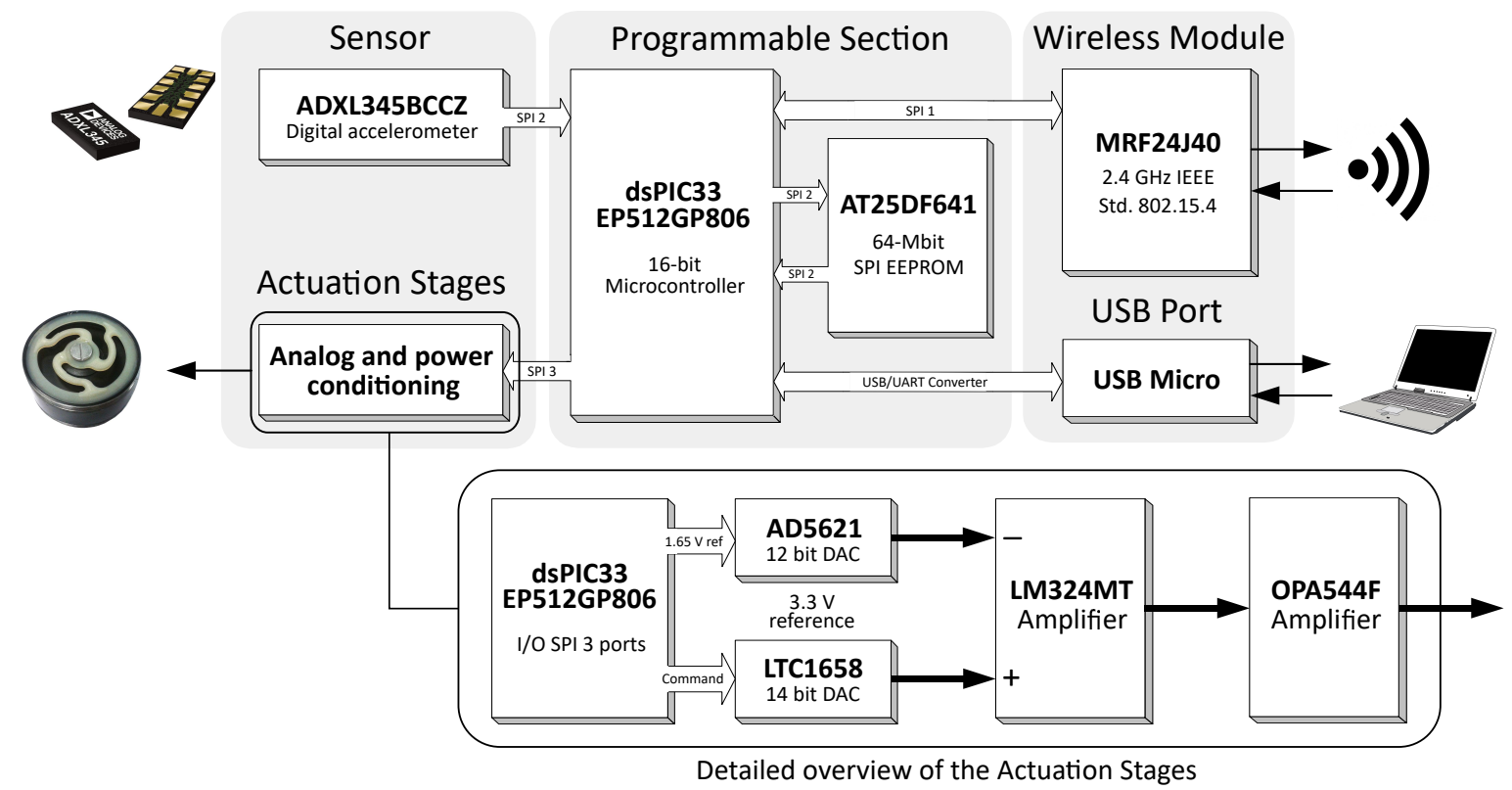

Figure 2: Block diagram which represents how the board is designed

transmission of commands in order to manage the operations of the board. Moreover, it is possible to read and acquire data from sensors.

\section{MATHEMATICAL MODEL}

\subsection{State-space system model}

The formulation of the mathemtical model of the system starts by considering the general motion equation (in the state variable matrix form) for a $n$-DOF controlled system:

$$
\underline{\dot{x}}(t)=[A] \underline{x}(t)+[B] \underline{u}(t)+[E] \underline{w}(t)
$$

where the matrices $A, B$ and $E$ are respectively the state matrix, the control input vector and the disturbance input vector. With sensors installed on the structure, the measurable system output $\underline{y}(t) \in \mathbb{R}^{p \times 1}$ is represented by a linear combination of the state of the system and the applied control forces:

$$
\underline{y}(t)=[C] \underline{x}(t)+[D] \underline{u}(t)+[F] \underline{w}(t)
$$

with $C \in \mathbb{R}^{p \times 2 n}, D \in \mathbb{R}^{p \times m}$ and $F \in \mathbb{R}^{p \times 1}$ ( $D$ and $F$ are matrices with all the terms equal to zero). In this notation, $n$ represents the degrees-of-freedom of the system, $m$ the number of regulators and $p$ the number of sensors. The continuous-time state-space model (Eq. 1) is now converted into the discrete-time domain with time step $\delta t$ using the $\mathrm{ZOH}$ discretization method:

$$
x(k+1)=\Phi x(k)+\Gamma u(k)+\Lambda w(k)
$$

\subsection{Linear Quadratic Regulator}

The development of Linear Quadratic Regulator allows to design an optimal control solution which minimizes the response of the structure minimizing the control effort. The trajectory of the control force $u$ is obtained by minimizing the scalar cost function $J$ :

$$
J(u)=\sum_{k=1}^{\infty}\left[x^{T}(k) Q x(k)+u^{T}(k) R_{c} u(k)+2 x^{T}(k) N u(k)\right]
$$


where $Q$ is a positive semi-definite matrix that penalizes the departure of system states from the equilibrium and $R_{c} \in \mathbb{R}^{p \times p}$ is a positive definite matrix that penalizes the control input. The matrices $Q$ and $R_{c}$ are often called the state cost matrix and the input cost matrix respectively. Considering the system equation:

$$
x(k+1)=\Phi x(k)+\Gamma u(k) \quad x \in \mathbb{R}^{n}, u \in \mathbb{R}^{p}
$$

with $x(0)=x_{0}$, to ensure the optimal control trajectory is physically feasible, minimization of the LQR cost function is constrained by Eq. 5 through the use of Lagrangian multipliers. The resulting linear control law can be written as:

$$
u(k)=-G x(k)
$$

where the matrix $G \in \mathbb{R}^{m \times 2 n}$ is the linear gain matrix defined as follows:

$$
G=\left(\Gamma^{T} S \Gamma+R_{c}\right)^{-1} \Gamma^{T} S \Phi
$$

\subsection{Kalman state estimation}

Looking at Eq. 6, the entire state vector $\underline{x}(k)$ is needed at each time step. Unfortunately, a measurement of the complete state of the system is not always available. Then, the measured output of the structure $\underline{y}(k)$ is exploited by a centralized controller to estimate the state $\underline{\hat{x}}$.

Kalman filter represents the most used tool for the state estimation. Considering the following linear timeinvariant (LTI) discrete-time system:

$$
\left\{\begin{array}{l}
x(k+1)=\Phi x(k)+\Gamma u(k)+\Lambda w(k) \\
y(k)=C x(k)+v(k)
\end{array}\right.
$$

the steady-state form of the estimator gain matrix $L$ is the following:

$$
L=\Phi P C^{T}\left(C P C^{T}+R_{v}\right)^{-1}
$$

where the matrix $P \in \mathbb{R}^{2 n \times 2 n}$ is the steady-state covariance matrix, which is the positive-definite solution of the Algebraic Riccati Equation. Note that, in each device, an independent Kalman estimator is designed by considering the same model of the system $\left(\Phi, \Gamma\right.$ and $\Lambda$ ), disturbance covariance $R_{w}$ and noise covariance $R_{v}$ (the only difference in the derivations is the output matrix $C$ ).

\section{SYNTHESIS OF THE CONTROLLER}

A commonly used approach in structural vibration control is the so called Skyhook damping technique. The aim of this approach is to increase the damping of the system by applying a force which is proportional to the velocity of the structure itself. The proposed solution, based on full-state feedback and Skyhook damping, requires the estimation of the remaining degrees-of-freedom of the system in order to properly work, since the number of measurements which is available is limited (it would be impossible to have a sensor for each free coordinate of the structure).

When a totally decentralized architecture is adopted and the Kalman filter is implemented, the quality of the estimated state will limit the performance of the controlled system, since only one measure is available to correct the state propagation. For this reason, the wireless communication channel is exploited and a state recovery strategy has been implemented, which is based on the idea that estimators exchange measurement data when estimation errors are larger than a given threshold. Each estimator compares its local measurement $y_{i}$ to the corresponding estimation of that degree-of-freedom $\bar{y}_{i}$, described by the relation $\bar{y}_{i}=C_{i} \underline{\underline{x}}_{F}$. In each device, the local measurement takes part in the controlled system during the update of the state estimate. The result of the update is the estimated state vector $\hat{x}(k)$ (which depends on $y(k)$ ) and the control signal $u(k)$ is finally computed through the relation reported in Eq. 6, substituting the real state vector $x(k)$ (not available) with the estimated one. Considering the $i^{t h}$ degree-of-freedom, the error between the measurement and the estimate is defined as:

$$
\epsilon=\left|\bar{y}_{i}-y_{i}\right|
$$


where $\epsilon \in \mathbb{R}^{p \times 1}$. When the error exceed a priori set threshold $\epsilon_{\max }$, the wireless sensor replaces the estimated state variable with the measured one. The measurement is then broadcast to the other devices within the network, in order to correct the corresponding estimations computed by other estimators in a synchronized way. In this manner, once the transmission is carried out, for each device in the system the updated state vector $\bar{x}_{u p}$ becomes:

$$
\bar{x}_{u p}=\left[\begin{array}{llllllllll}
\dot{\bar{x}}_{F, 1} & \cdots & \dot{x}_{F, i} & \cdots & \dot{\bar{x}}_{F, n} & \bar{x}_{F, 1} & \cdots & \bar{x}_{F, i} & \cdots & \bar{x}_{F, n}
\end{array}\right]^{T}
$$

So, the calculation of the control force for that time instant is performed by using the updated state vector $\bar{x}_{u p}$ instead of $\hat{x}$, left-multiplied by the LQR gain matrix $G \in \mathbb{R}^{1 \times 2 n}$ :

$$
u=-G \bar{x}_{u p}
$$

To sum up, each device estimates the whole state of the sytem starting from the only local measurement which is available; the state of the system consists in velocities and displacements of the free coordinates and the local measurement corresponds to the vertical acceleration of the device coordinate (which is properly integrated to obtain the velocity value). The state recovery algorithm aims to improve the quality of the estimation; considering $n$ stand-alone devices, the state components which will be reconstructed with a higher precision will be the ones related to the $n$ devices locations. Therefore, as the number of devices increases, the estimated state will be one step closer to the real one.

\subsection{Reduced model}

As well known, when one deals with mathematical models of the systems, their dynamics are never fully taken into account; for this reason, the best thing to do is to define a reduced model which can accurately describe the dynamics of the system in the frequency range of interest. It can be determined through Finite Element Analysis (FEA) or experimental modal identification techniques. ${ }^{29,30}$ This kind of approach allows to greatly simplify the model of the system by reducing the number of degrees-of-freedom to a small set of decoupled modal coordinates, also obtaining a lower computational effort. The reduced model is now derived, on which all the control algorithms will be based. Then, the observer and control gain matrices will be computed.

In this work the reduced model will be made of only three modes. In particular, they are the first three modes of the beam which will be used into the experimental phase. The choice of the reduced model size and, therefore, of the vibration modes that will define it, will be explained later. So, $n_{R}=3$ is the number of modes of the reduced model and $n=90$ is the number of degrees-of-freedom of the system. Then, the $n_{R}$ motion equations in modal coordinates can be written as:

$$
[\tilde{M}] \underline{\ddot{q}}_{R}(t)+[\tilde{R}] \underline{\dot{q}}_{R}(t)+[\tilde{K}] \underline{q}_{R}(t)=\left[\tilde{\Phi}\left(\underline{\xi}_{d}\right)\right]^{T} \underline{F}_{d}(t)+\left[\tilde{\Phi}\left(\underline{\xi}_{c}\right)\right]^{T} \underline{F}_{c}(t)
$$

where $q_{R}$ is the $n_{R} \times 1$ vector of the modal displacements and $\tilde{M}, \tilde{R}$ and $\tilde{K}$ are the $n_{R} \times n_{R}$ reduced modal matrices. $\tilde{\Phi}\left(\underline{\xi}_{d}\right)$ and $\tilde{\Phi}\left(\underline{\xi}_{c}\right)$, instead, are the transposed reduced modal matrices evaluated at the points of application of the disturbance forces $\left(\xi_{d}\right)$ and the control forces $\left(\xi_{c}\right)$, respectively. Using a modal approach it is simple to verify the controllability of the system by observing the Lagrangian components of the control forces with respect to the modal coordinates: ${ }^{31}$ referring to the general equation of the $i^{t h}$ controlled mode, the following relation must be verified:

$$
\underline{\tilde{\Phi}}_{i}\left(\underline{\xi}_{c}\right)^{T} \underline{F}_{c}(t) \neq 0
$$

for $i=1, \cdots, n_{c}$. Physically, this condition is achieved by placing, for each controlled mode, at least one actuator in a nonnodal position for the considered mode.

Modal velocities $\dot{q}_{R}$ can be calculated using $m$ sensors measuring physical displacements, velocities or acceleration (in this case the information coming from sensors are accelerations, so it is needed an integration). Considering the reduced model, the relationship between physical and modal velocities is:

$$
\underline{\dot{x}}(t)=\left[\tilde{\Phi}\left(\underline{\xi}_{m}\right)\right] \underline{\dot{q}}_{R}(t)
$$


where $\tilde{\Phi}\left(\underline{\xi}_{m}\right)$ is an $m \times n_{R}$ matrix, obtained from the modal matrix of the reduced model, evaluated at points where sensors are located $\left(\underline{\xi}_{m}\right)$.

Now, thanks to the information just obtained, it is possible to derive the modal state-space form of the system starting from the physical one described in Eq. 1:

$$
\underline{\dot{q}}_{s s}(t)=[\tilde{A}] \underline{q}_{s s}(t)+[\tilde{B}] \underline{u}(t)+[\tilde{E}] \underline{w}(t)
$$

where the matrices $\tilde{A}, \tilde{B}$ and $\tilde{E}$ are now defined considering only the modal contributions. The modal state vector of the reduced model $\underline{q}_{s s}$ is written as:

$$
\underline{q}_{s s}(t)=\left[\underline{\dot{q}}_{R}(t) \quad \underline{q}_{R}(t)\right]^{T}
$$

The measurable system output $\underline{y}(t) \in \mathbb{R}^{p \times 1}$ is still defined as in Eq. 2:

$$
\underline{y}(t)=[\tilde{C}]_{\underline{q}_{s s}}(t)
$$

This relation allows to switch directly from the modal coordinates to the physical coordinates of the system of which the measures (in this case only velocity) are known. The matrix $\tilde{C}$, which is the conversion/extraction matrix, is defined as:

$$
\tilde{C}=\left[\begin{array}{ll}
{\left[\tilde{\Phi}\left(\underline{\xi}_{m}\right)\right]} & {[0]}
\end{array}\right] \in \mathbb{R}^{m \times 2 n_{R}}
$$

Then, once all the necessary matrices have been defined, it is possible to proceed with the derivation of the Kalman filter. As introduced before, the idea is to develop an independent Kalman estimator on each wireless sensor which works only with its own local measurement. The only known input of the Kalman filter is related to its control force, generated by the full-state feedback driven by the optimal Linear Quadratic Regulator. The control forces applied to the structure by the other devices are detected by each estimator as an additional disturbance, and the estimate is adapted as well as it is done for external disturbances.

In order to calculate the observer gain matrix $\tilde{L}$, the reduced model just described must be discretized; therefore, the state-space matrices of the reduced model $\tilde{A}, \tilde{B}$ and $\tilde{E}$ become respectively $\tilde{\Phi}, \tilde{\Gamma}$ and $\tilde{\Lambda}$. The matrix $\tilde{L}$ is then obtained by substituting the modal matrices into the Eq. 9:

$$
\tilde{L}=\tilde{\Phi} \tilde{P} \tilde{C}^{T}\left(\tilde{C} \tilde{P} \tilde{C}^{T}+R_{v}\right)^{-1} \in \mathbb{R}^{2 n_{R} \times 1}
$$

As regards the formulation of the optimal Linear Quadratic Regulator, instead, a different way has been followed. To calculate the observer gain matrix, the Riccati equation has been solved by considering the system already in modal coordinates; in the case of the controller gain matrix, on the other hand, the physical coordinates of the system have been first considered. This is due to the fact that, since it is not a modal control, it is easier to formulate the weights matrix $\mathrm{Q}$ referring directly on the physical degrees-of-freedom of the system.

Considering that the resulting control signal will be proportional to each of these coordinates, a good way of proceeding could be:

1. to assign the highest weight to the coordinate of which the measurement is known;

2. to assign a lower weight to the other vertical coordinates;

3. to assign null weight to the horizontal and rotational coordinates (and also to the degrees-of-freedom related to the suspended masses of inertial actuators).

Of course, the weight assignment set is referred to the velocities, because the weight related to the displacements is null for each coordinate. The difference in weights between the local coordinate of each device and the others is due to the fact that the estimate of the local one is the most precise, since its true measure is known, while the 


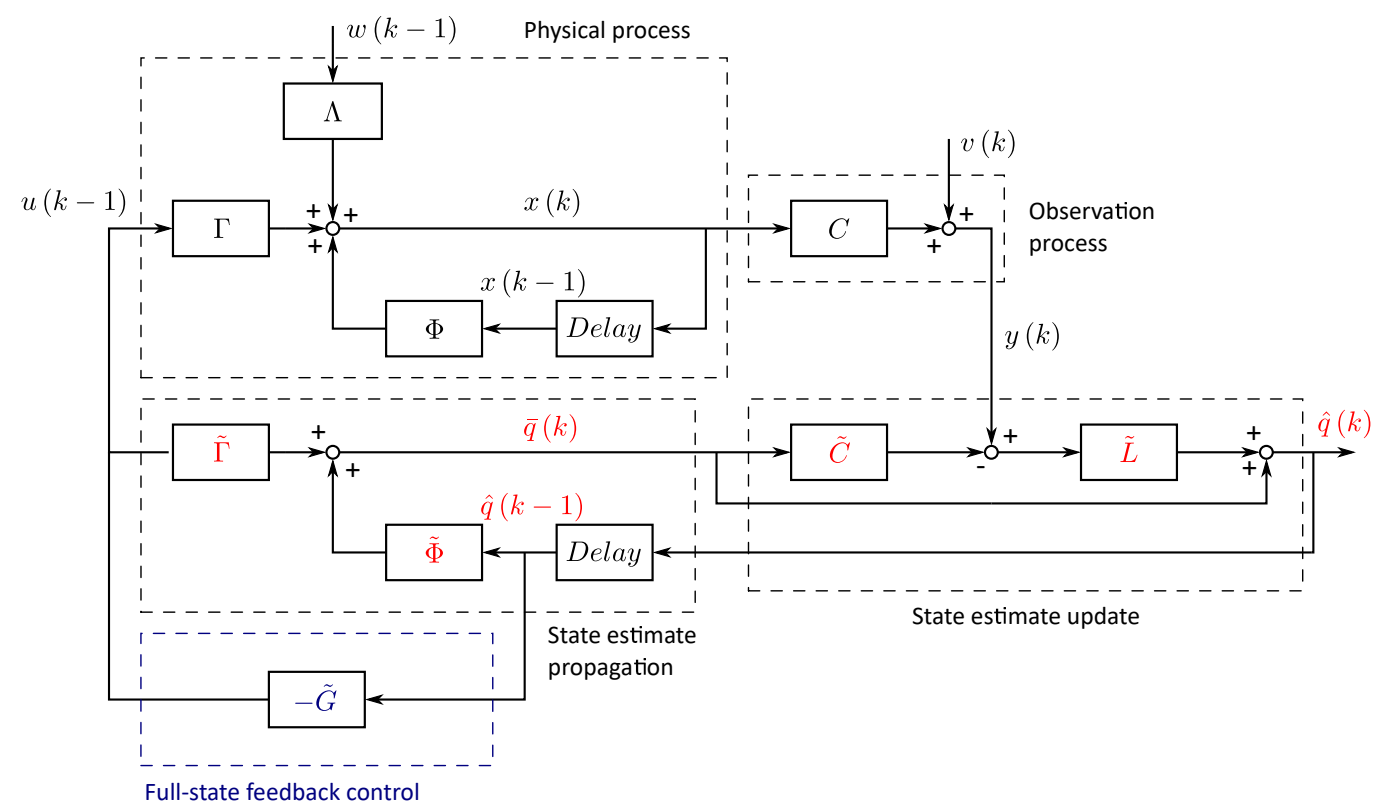

Figure 3: Dynamic system, modal state estimator and regulator block diagrams

estimates of the other vertical coordinates are purely mathematical. The weight assigned to the latter, however, is not too low, since with the state recovery it is possible to improve the quality of the estimated state. Therefore, the matrix of the weights $Q$ will take a form like the following:

$$
Q=\left[\begin{array}{cc}
{\left[Q_{\dot{x}}\right]} & {[0]} \\
{[0]} & {[I]}
\end{array}\right] \in \mathbb{R}^{2 n \times 2 n}
$$

where $Q_{\dot{x}}$ is the diagonal weights matrix of the velocities just described. The control effort weights matrix $R_{c}$ has been set equal to 1 ; this is a normalized form, since in the minimization of $J$ what matter is the relative weight of the matrix $Q$ and $R$. At this point, the control Algebraic Riccati Equation is solved and then the controller gain matrix $G$ is calculated as shown in Eq. 7. Finally, the last step concerns the conversion of the matrix $G$ from the domain of the physical coordinates to that of the modal coordinates (since the estimator works in modal coordinates). This step is carried out by means of the transformation matrix $\Lambda_{q x}$ defined as follows:

$$
\tilde{G}=[G]\left[\Lambda_{q x}\right]=[G]\left[\begin{array}{cc}
{\left[\Phi_{R}\right]} & {[0]} \\
{[0]} & {\left[\Phi_{R}\right]}
\end{array}\right] \in \mathbb{R}^{1 \times 2 n_{R}}
$$

with $\Lambda_{q x} \in \mathbb{R}^{2 n \times 2 n_{R}}$. So, the command signal is now calculated directly from the modal state vector as:

$$
u(k)=-\tilde{G} q_{s s}(k)
$$

In Fig. 3 is shown the complete block diagram representing the model of the real system, the modal state estimator and the Linear Quadratic Regulator.

When in a device the state recovery is triggered by the device itself or by the others present in the network, the modal state vector must be temporarily converted into the physical state vector through the transformation matrix $\Lambda_{q x}$, in order to replace the estimate of that specific coordinate $\dot{\bar{x}}_{F, i}$ with its corresponding measure $\dot{x}_{F, i}$ (as shown in Eq. 11). Once the measurement has been replaced, the physical state vector is re-converted into the modal one by means of the matrix $\Lambda_{x q}$, which is defined as:

$$
\Lambda_{x q}=\left[\begin{array}{cc}
{\left[\Phi_{R, i n v}\right]} & {[0]} \\
{[0]} & {\left[\Phi_{R, i n v}\right]}
\end{array}\right] \in \mathbb{R}^{2 n_{R} \times 2 n}
$$

where $\Phi_{R, i n v}$ is the $n_{R} \times n$ complementary reduced modal matrix, which is derived from the inverse modal matrix $\Phi^{-1}$ by means of an extraction matrix that, in this case, picks up only the three rows corresponding to the selected vibration modes (which is the transposed of the extraction matrix used to obtain $\Phi_{R}$ ). 


\section{NUMERICAL ANALYSIS}

To analyze the proposed solution, some numerical analyzes have been performed using the algorithm described in the previous section. The aim of this process is to verify the performance of the modal estimator described before and, mainly, to understand how much the state recovery could be advantageous for the improvement of the quality of the estimated state vector. The behavior of the controlled system will be investigated in the experimental phase, because once the improvement in the accuracy of the estimate is demonstrated, the regulator will be able to properly control the system, with no need to prove it numerically.

The system which has been used to perform the numerical analysis is a steel beam with a rectangular cross section, clamped on its ends; this structure has been used also in the experimental tests (Section 6). The parameters of the beam are summarized in Table 1. Two physical system configurations have been considered: in the first one, only two wireless sensors are involved (which are called Device 1 and Device 2), while in the second one four wireless sensors are considered (Device 1,2,3,4). In both configurations the disturbance force in generated by means of an inertial actuator like the ones used for smart dampers (which is called Shaker 1). Considering the modal approach which has been presented, the arrangement of the wireless sensors on the structure has a very high influence on the quality of the estimator output. Since the reduced model of the system has been developed using only three vibration modes and since the number of wireless sensors available is very low, a wrong positioning of them with respect to the shape of the modeled modes would generate a badly conditioned state matrix. A similar situation could significantly worsen the performance of the estimator. Therefore, in order to simplify the management of this kind of reasoning, the model has been set up in a certain way and all tests have been carried out under the same conditions. The disturbance action generated by Shaker 1 has been set in order to excite the resonance frequency of mode III $(94.72 \mathrm{~Hz})$. In Table 2 are reported the natural frequencies $f_{i}=\omega_{i} /(2 \pi)$ of the system obtained both numerically and experimentally through the modal analysis; for this study, vibration modes up to the fifth have been considered (labeled from I to V). The resonance frequencies related to inertial actuators dynamics have not been considered. Then, wireless sensors have been positioned as a function of the nonnodal positions of all modeled modes, focusing on the one excited by the disturbance force. The aim was to satisfy Eq. 14, which is relatively easy for simple systems (like the one used), but which may become very difficult for complex systems. To overcome this problem, the developed device can perform a preliminary modal identification in order to check the controllability of the system; if the device is badly located, the board will communicate the information to the operator and then it will be possible to move the actuator in a better position. In Fig. 4 is shown the model of the system, which represents the physical

\begin{tabular}{llll}
\hline Parameter & Symbol & Value & Units \\
\hline Beam length & $L$ & 2.37 & $\mathrm{~m}$ \\
Beam height & $h$ & 0.02 & $\mathrm{~m}$ \\
Beam width & $w$ & 0.08 & $\mathrm{~m}$ \\
Cross-section & $A$ & 0.016 & $\mathrm{~m}^{2}$ \\
Young modulus & $E$ & 190 & $\mathrm{GPa}$ \\
Density & $\rho$ & 7900 & $\frac{\mathrm{kg}}{\mathrm{m}^{3}}$ \\
Inertia moment & $J$ & $5.452 \times 10^{-8}$ & $\mathrm{~m}^{4}$ \\
Mass & $m$ & 12.73 & $\frac{\mathrm{kg}}{\mathrm{m}}$ \\
\hline
\end{tabular}

Table 1: Structural parameters of the beam

\begin{tabular}{lllllll}
\hline \multicolumn{2}{c}{ Mode } & I & II & III & IV & V \\
\hline \multirow{2}{*}{$f_{i}[\mathrm{~Hz}]$} & Num & 16.84 & 49.39 & 94.96 & 158.95 & 235.27 \\
& Exp & 17.02 & 49.08 & 94.72 & 159.11 & 235.51 \\
\hline
\end{tabular}

Table 2: Resonance frequencies of the system obtained both numerically and experimentally 


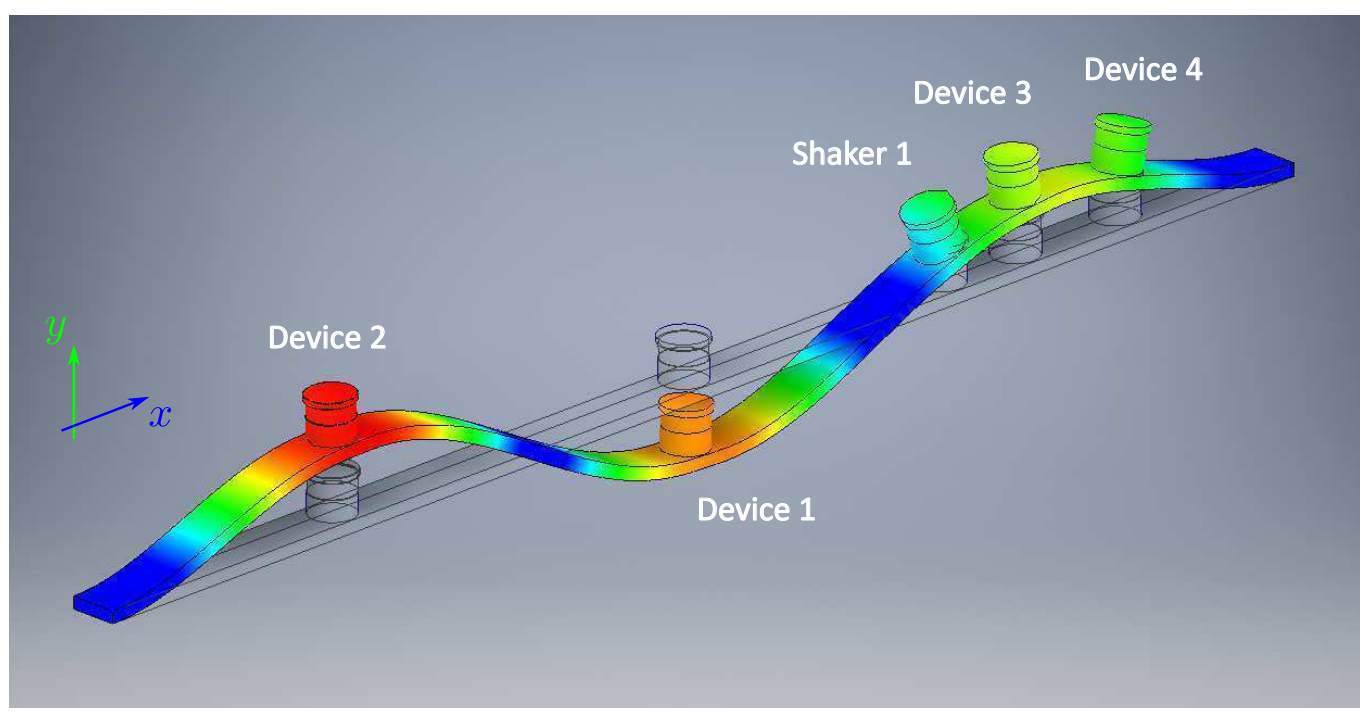

Figure 4: Model of the system used for the numerical analysis, showing the arrangement of devices and the shape of the third vibration mode

\begin{tabular}{lllllll}
\hline & Device 1 & Device 2 & Device 3 & Device 4 & Shaker 1 & Units \\
\hline Position & 1.19 & 0.48 & 1.82 & 2.05 & 1.66 & $\mathrm{~m}$ \\
\hline
\end{tabular}

Table 3: Positions of devices (wireless sensors and shaker) along the beam

arrangement of devices with respect to the beam (the system is excited at the frequency of mode III); devices locations placed on the beam are summarized in Table 3 .

As explained before, the state recovery is triggered in each device when the error between the estimate and the local measurement exceeds a certain threshold $\left(\epsilon_{\max }\right)$; therefore, by varying the value of this threshold, the frequency with which the data are exchanged within the network can increase or decrease. Starting from the maximum value of the error threshold $\epsilon_{\max }$ which triggers the state recovery, several simulations have been carried out, decreasing the value of the threshold each time (the same value of the threshold has been used for all devices). This procedure has been carried out both for the two-devices and four-devices configurations and, in both cases, the same set of error thresholds has been used. The two most significant types of data which have been obtained concern, for each wireless sensor, the average frequency of data transmission via the wireless channel $f_{\text {transm }}$ and $\xi_{a v}$, which is the average of the error $\xi$ between the estimate and the exact value of the velocity (the exact value in the real case is unknown). For each value of the threshold $\epsilon_{\max }$ it is thus possible to obtain the corresponding average transmission frequency and average error of the estimate; the curves that describe the trend of these two parameters as a function of the error threshold have been derived for both configurations.

In Fig. 5 is represented the steps sequence performed during the numerical analysis, which are described as follows. Note that these figures are related only to Device 1 (the procedure is the same for other devices).

- Step 1: in Fig. 5a are shown the trends of the errors on the estimate as a function of the error threshold, which have been obtained as described before. The two curves represent the two-devices and four-devices configurations defined as $\xi_{a v, 2 d e v}=f_{2 d e v}\left(\epsilon_{\max }\right)$ and $\xi_{a v, 4 d e v}=f_{4 d e v}\left(\epsilon_{\max }\right)$ (in blue and red respectively).

- Step 2: the curve in Fig. 5b, instead, represents the function $\xi_{a v, d i f f}=f_{\text {diff }}\left(\epsilon_{\max }\right)$ defined as $\xi_{a v, \text { diff }}=$ $\xi_{a v, 2 d e v}-\xi_{a v, 4 d e v}$. This plot was created in order to choose, according to some criteria, the error threshold values to use to implement the state recovery algorithm.

- Step 3: two error thresholds have been chosen. The first one ( $\epsilon_{\max , 1}$, depicted in dotted line) is related to the maximum value of the curve $\xi_{a v, d i f f}$; this choice represents the maximum efficiency condition, that is 


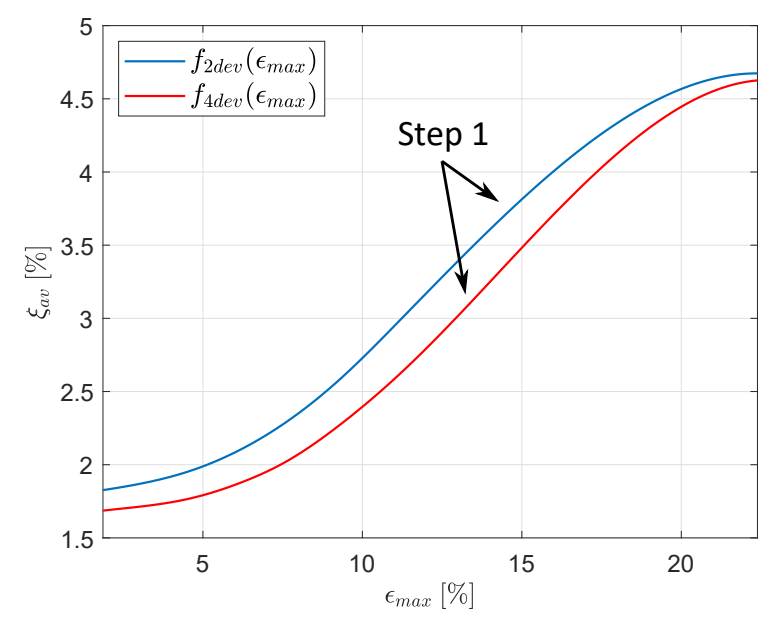

(a)

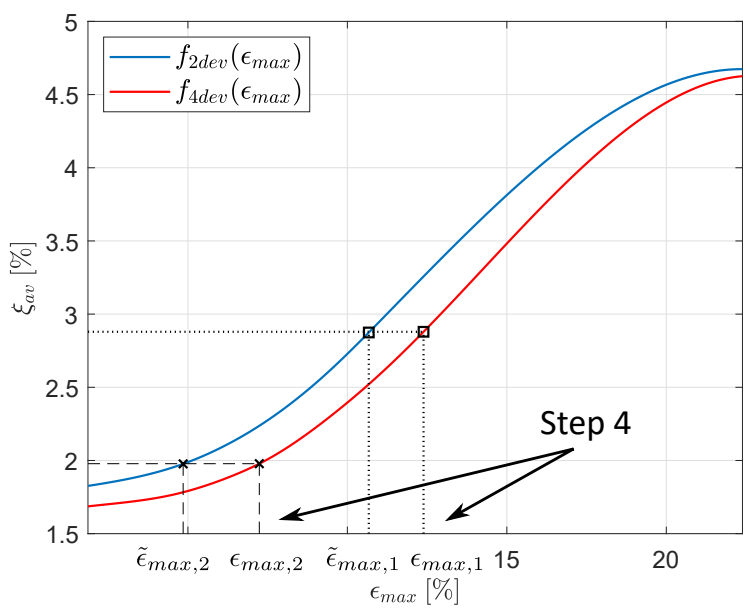

(c)

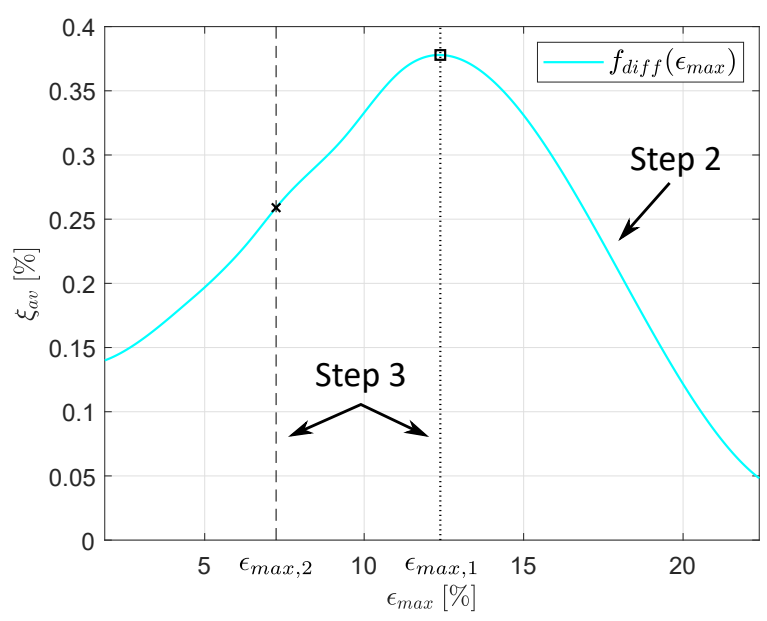

(b)

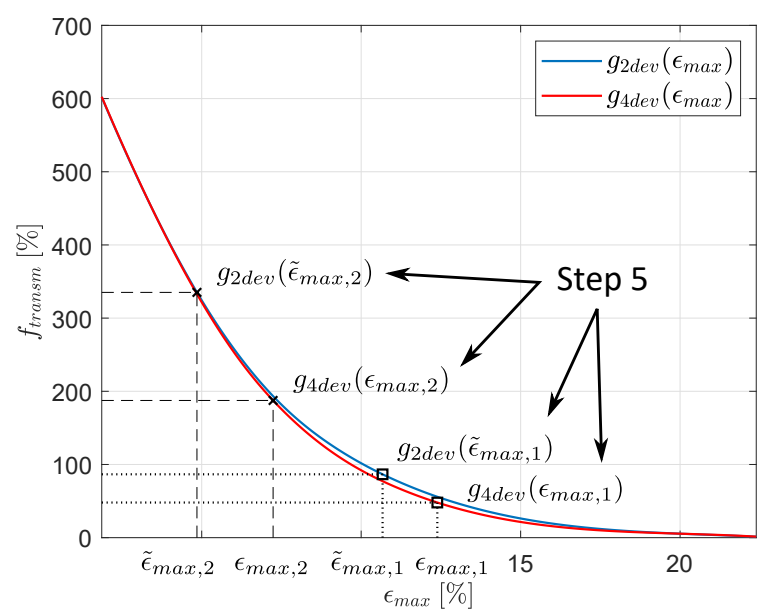

(d)

Figure 5: Steps sequence performed during the numerical analysis

the condition in which the maximum relative performance increase is obtained in using four devices instead of two. The second one $\left(\epsilon_{\max , 2}\right.$, depicted in dashed line) is related to the average value of the curve $\xi_{a v, d i f f}$ for $\epsilon_{\max } \leq \epsilon_{\max , 1}$; this choice represents a trade-off between performance level and computational effort. $\epsilon_{\max , 1}$ and $\epsilon_{\max , 2}$ are used in the four-devices configuration.

- Step 4: starting from $\epsilon_{\max , 1}$ and $\epsilon_{\max , 2}$ obtained in Step 3, the two equivalent error thresholds $\tilde{\epsilon}_{\max , 1}$ and $\tilde{\epsilon}_{\max , 2}$ in the two-devices configuration have been obtained by using the two curves described in Fig. $5 \mathrm{a}$ as follows:

$$
\left\{\begin{array}{l}
\tilde{\epsilon}_{\max , 1}=f_{2, \operatorname{dev}}^{-1}\left(f_{4, \operatorname{dev}}\left(\epsilon_{\max , 1}\right)\right) \\
\tilde{\epsilon}_{\max , 2}=f_{2, \operatorname{dev}}^{-1}\left(f_{4, \operatorname{dev}}\left(\epsilon_{\max , 2}\right)\right)
\end{array}\right.
$$

In the two-devices configuration, these thresholds guarantee the same average error $\xi_{a v}$ obtained in the other configuration; in this way, it can be seen how much the error threshold has to be decreased in order to guarantee the same accuracy on the estimate.

- Step 5: in Fig. 5d are shown the trends of average frequencies of data transmission as a function of 
the error threshold. The two curves represent the two-devices and four-devices configurations defined as $f_{\text {transm,2dev }}=g_{2 d e v}\left(\epsilon_{\max }\right)$ and $f_{\text {transm,4dev }}=g_{4 d e v}\left(\epsilon_{\max }\right)$ (in blue and red respectively). The two set of thresholds $\left(\epsilon_{\max , 1}, \tilde{\epsilon}_{\max , 1}\right)$ and $\left(\epsilon_{\max , 2}, \tilde{\epsilon}_{\max , 2}\right)$ have been used to obtain the corresponding set of transmission frequencies for the two cases (maximum efficiency and midpoint):

$$
\left\{\begin{aligned}
f_{\text {transm }, 2 \text { dev }, 1} & =g_{2 \operatorname{dev}}\left(\epsilon_{\text {max }, 1}\right) \\
f_{\text {transm }, 4 \text { dev }, 1} & =g_{4 d e v}\left(\tilde{\epsilon}_{\text {max }, 1}\right) \\
f_{\text {transm }, 2 d e v, 2} & =g_{2 d e v}\left(\epsilon_{\text {max }, 2}\right) \\
f_{\text {transm }, 4 \text { dev }, 2} & =g_{4 d e v}\left(\tilde{\epsilon}_{\text {max }, 2}\right)
\end{aligned}\right.
$$

To quantify the difference between the two configurations, in Tables 4 and 5 are reported the numerical values obtained with Device 1 and Device 2 respectively (only data coming from Device 1 and 2 have been chosen as they belong to both configurations). For $\xi_{a v}$ and $f_{\text {transm }}$ there is a distinction between the theoretical values $(\mathrm{TH})$, i.e. obtained from the plots, and those obtained from the simulations (SIM).

Looking at the "max efficiency" column, one can note that the average transmission frequencies are quite low. For this reason, in Step 3 also the threshold $\epsilon_{\max , 2}$ was chosen; by lowering the error threshold, a higher accuracy in the estimation is ensured, without overloading the wireless channel (in spite of the increase in transmission frequency). The ratio of errors and frequencies between one case and the other is not the same, i.e. a smaller reduction of the error with respect to the increase in frequency is obtained. However, the frequency is kept very limited and good results in terms of accuracy of the estimate are guaranteed.

In Fig. 6 are shown the results obtained without (6a) and with (6b) the update of the state vector with the four-devices configuration (Device 1 data); in each plot the measured, estimated and real velocities are reported, which are depicted in blue, red and green respectively. Looking at the two figures it can be noted the improvement in the estimation of the velocity obtained with to the state recovery approach. In Fig. 7, instead, is represented the trend of the error on the estimate $\xi$ without (7a) and with (7b) state recovery for Device 1, considering the "midpoint" case. Considering the average of errors in both cases, when the state recovery is applied the algorithm ensures a reduction of the mean error of about $42 \%$.

\begin{tabular}{|c|c|c|c|c|c|c|}
\hline \multirow{2}{*}{\multicolumn{2}{|c|}{ Updates }} & \multicolumn{2}{|c|}{ Max efficiency } & \multicolumn{2}{|c|}{ Midpoint } & \multirow[t]{2}{*}{ Units } \\
\hline & & 2 & 4 & 2 & 4 & \\
\hline \multicolumn{2}{|c|}{$\epsilon_{\max }$} & 10.6716 & 12.3881 & 4.8507 & 7.2388 & $\%$ \\
\hline \multirow{2}{*}{$\xi_{a v}$} & TH & 2.8745 & 2.8792 & 1.9766 & 1.9781 & \multirow{2}{*}{$\%$} \\
\hline & SIM & 2.8703 & 2.8784 & 1.9737 & 1.9772 & \\
\hline \multirow{2}{*}{$f_{\text {transm }}$} & TH & 86.5799 & 47.9978 & 335.1500 & 187.4737 & \multirow{2}{*}{$\mathrm{Hz}$} \\
\hline & SIM & 86.1589 & 47.7596 & 335.0517 & 186.5468 & \\
\hline
\end{tabular}

Table 4: Simulations results: Device 1

\begin{tabular}{|c|c|c|c|c|c|c|}
\hline \multirow{2}{*}{\multicolumn{2}{|c|}{ Updates }} & \multicolumn{2}{|c|}{ Max efficiency } & \multicolumn{2}{|c|}{ Midpoint } & \multirow[t]{2}{*}{ Units } \\
\hline & & 2 & 4 & 2 & 4 & \\
\hline \multicolumn{2}{|c|}{$\epsilon_{\max }$} & 10.6716 & 12.3881 & 4.4776 & 7.2388 & $\%$ \\
\hline \multirow{2}{*}{$\xi_{a v}$} & TH & 2.8990 & 2.9006 & 1.9793 & 1.9757 & \multirow{2}{*}{$\%$} \\
\hline & SIM & 2.9012 & 2.9037 & 1.9781 & 1.9776 & \\
\hline \multirow{2}{*}{$f_{\text {transm }}$} & TH & 89.9056 & 50.7291 & 339.2500 & 181.2499 & \multirow{2}{*}{$\mathrm{Hz}$} \\
\hline & SIM & 89.9167 & 50.7225 & 339.3684 & 181.3096 & \\
\hline
\end{tabular}

Table 5: Simulations results: Device 2 


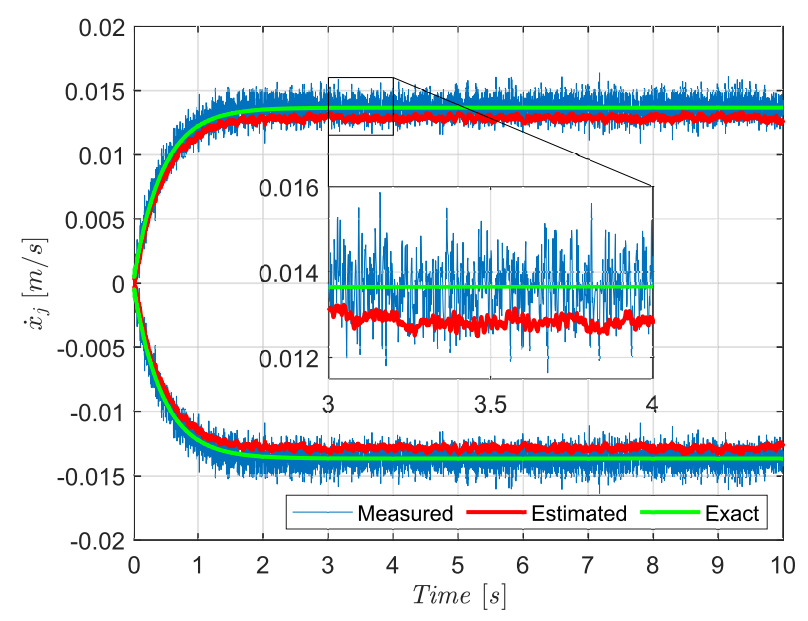

(a)

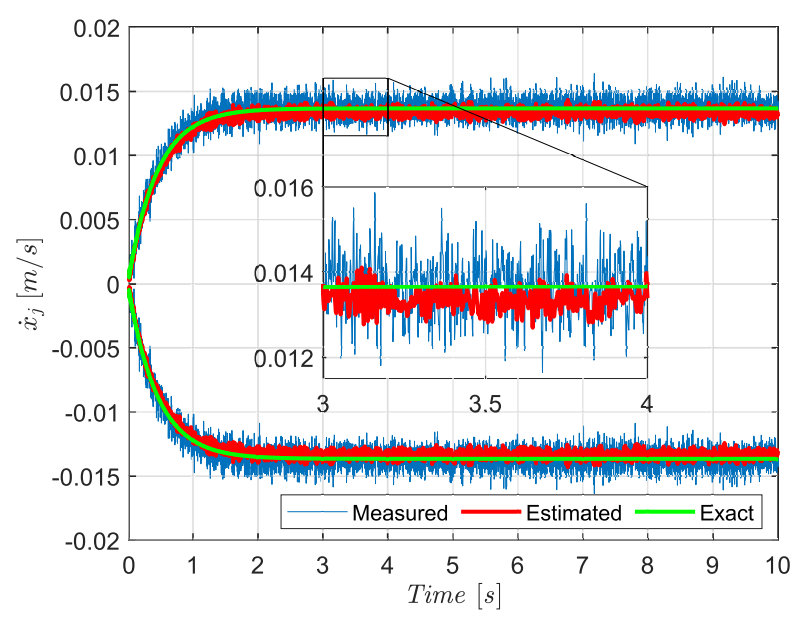

(b)

Figure 6: Comparison between the measured data (blue line), the estimated (red line) and the exact values (green line) of the velocity without (a) and with (b) the wireless state update

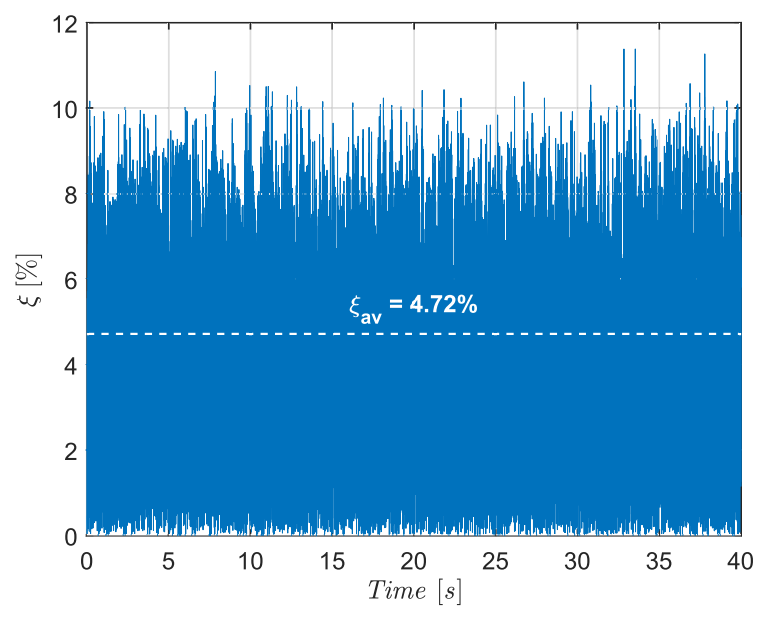

(a)

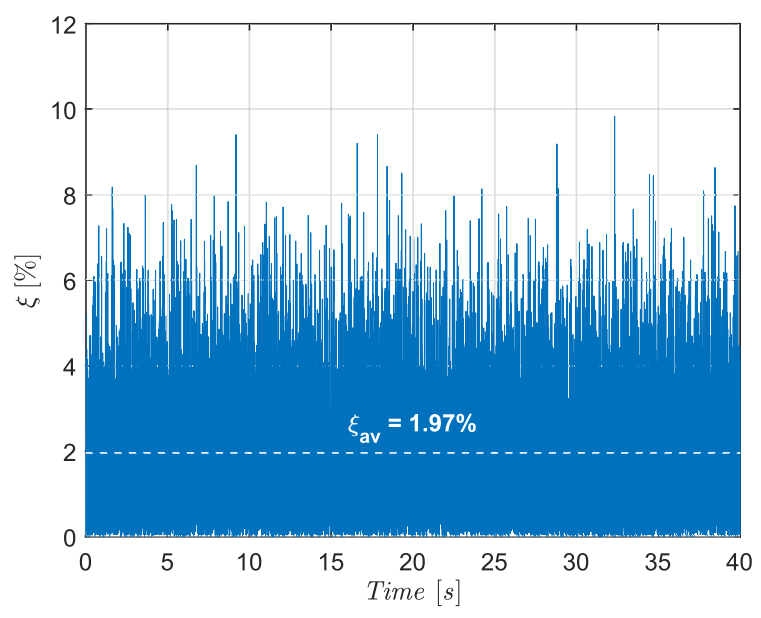

(b)

Figure 7: Error of the local velocity estimate with respect to its real value without (a) and with (b) the wireless state update

The number of devices involved in the state recovery algorithm is the variable that most affects the performance of the entire system. The choice of this number results from a trade-off between quality of the state estimate and data traffic within the network. Fig. $6 \mathrm{~b}$ proves that four devices represents a good compromise to obtain good performance both in terms of quality and saturation of the wireless channel. However, the solution is not trivial, because many variables such as the features of the system and hardware performance could affect the final result.

\section{EXPERIMENTAL RESULTS}

In order to verify the performance of the developed control strategy in suppressing vibration, some tests have been performed using an experimental test rig like the model described in Section 5. On the beam are placed two 


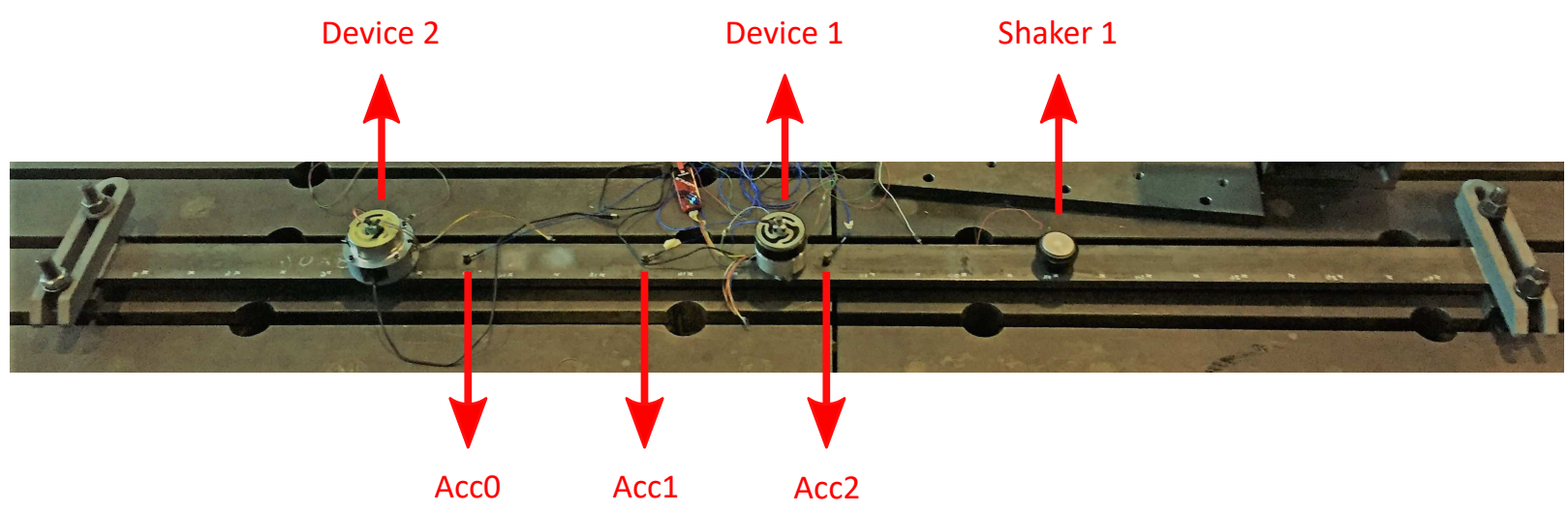

Figure 8: Experimental test rig

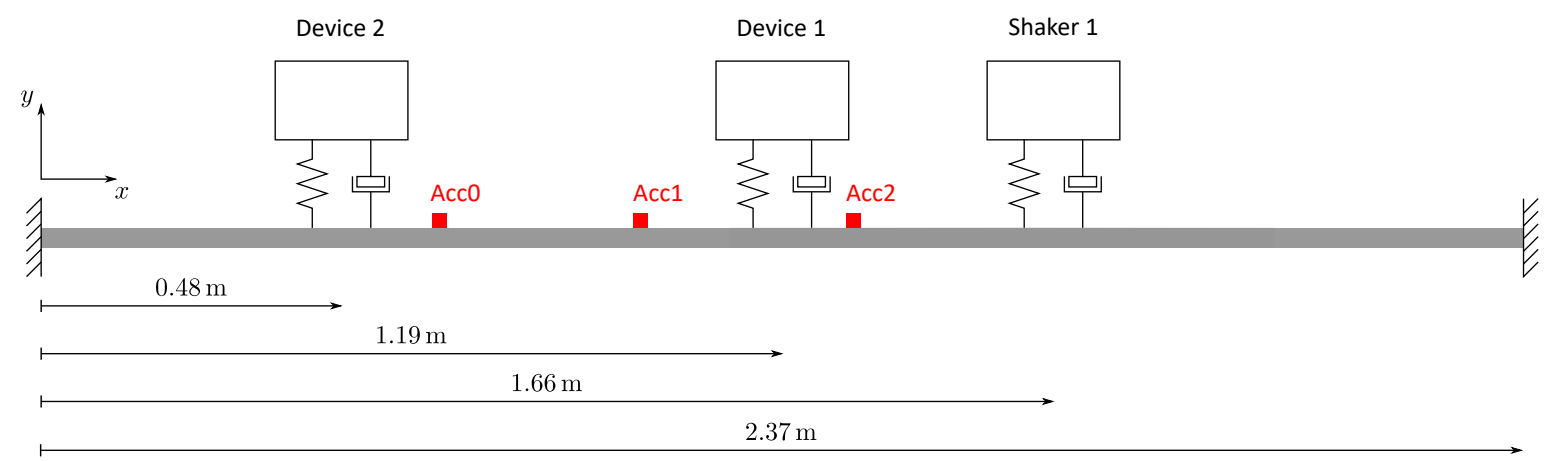

Figure 9: Configuration scheme of the beam

stand-alone devices (Device 1 and Device 2) and a shaker (Shaker 1), as shown in Fig. 8 and Fig. 9; moreover, three piezoelectric accelerometers $(A c c 0, A c c 1$ and $A c c 2)$ are mounted on the structure in order to the detect the vibration state in a global way (only for data sampling, they are not involved in the control action). The position along the beam of smart dampers, shaker and analog accelerometers is reported in Table 6 .

As described in Section 4.1, the definition of a reduced model of the system also comes from the need to limit the computational effort as much as possible. Three vibration modes have been chosen, which are modes I, II and III $(17.02 \mathrm{~Hz}, 49.08 \mathrm{~Hz}$ and $94.72 \mathrm{~Hz})$, representing the first three resonances of the structure related to the nature of the beam. In this case, three modal coordinates represent a good trade-off between bandwidth and computational cost. A greater number of coordinates could be considered by improving the capabilities of the microcontroller. Considering the limited number of modal degrees-of-freedom, those related to inertial actuators are not considered; in this way the reduced model is guaranteed to be valid over a wider frequency range. Moreover, the contribution of information which these modes provide to the model is very low, so it is preferable to build the model with the most relevant modal components.

This experimental phase, therefore, has been focused on the implementation of the Kalman filter and the

\begin{tabular}{llll}
\hline Device & Device 1 & Device 2 & Shaker 1 \\
\hline Position [m] & 1.19 & 0.48 & 1.66 \\
\hline Device & Acc0 & Acc1 & Acc2 \\
\hline Position [m] & 0.63 & 0.95 & 1.26 \\
\hline
\end{tabular}

Table 6: Summary table of the devices (actuators and sensors) positioned on the beam 


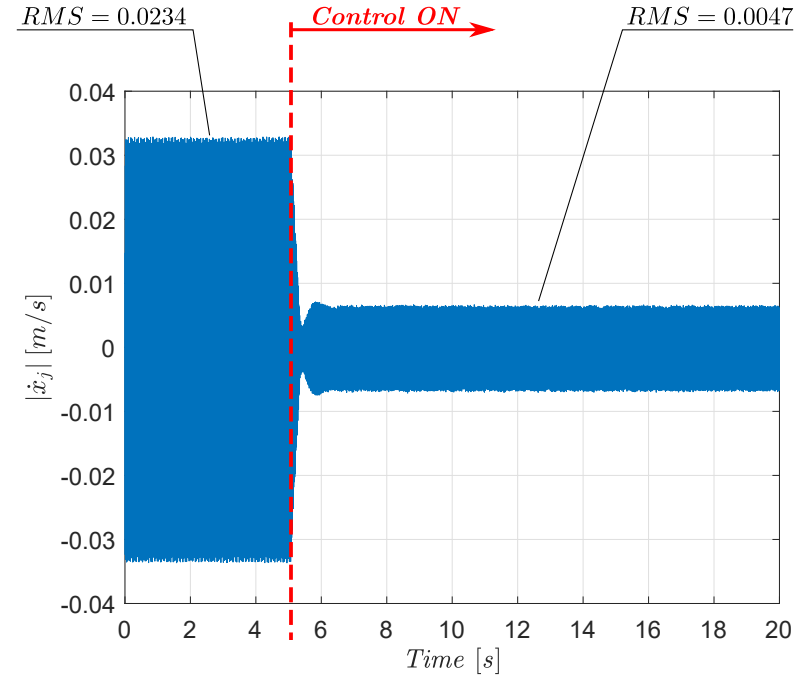

(a)

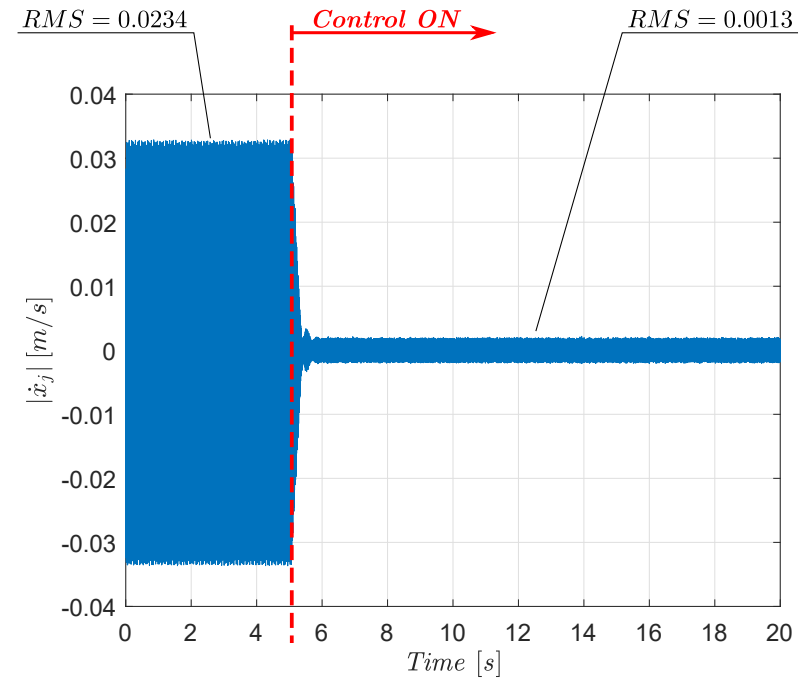

(b)

Figure 10: Comparison between the results (velocity) of the controlled system obtained experimentally without (a) and with (b) the state recovery - Mode III

Linear Quadratic Regulator on both wireless sensors, coupled with the state recovery algorithm. The experimental tests are related to the control of mode III and the disturbance is a tone whose frequency is tuned on the eigenfrequency of that mode $(94.72 \mathrm{~Hz})$. The results obtained in this case are shown in Fig. 10, in which are depicted the measured velocities related to the controlled system without (Fig. 10a) and with (Fig. 10b) the recovery of the state. The control action, in the second case, has a very high efficiency: the RMS value of the velocity is in fact reduced by $94 \%$ with respect to the uncontrolled case, considering both devices involved into the control (in the first case, instead, the reduction is about 80\%). In Fig. 11 is shown the frequency spectrum

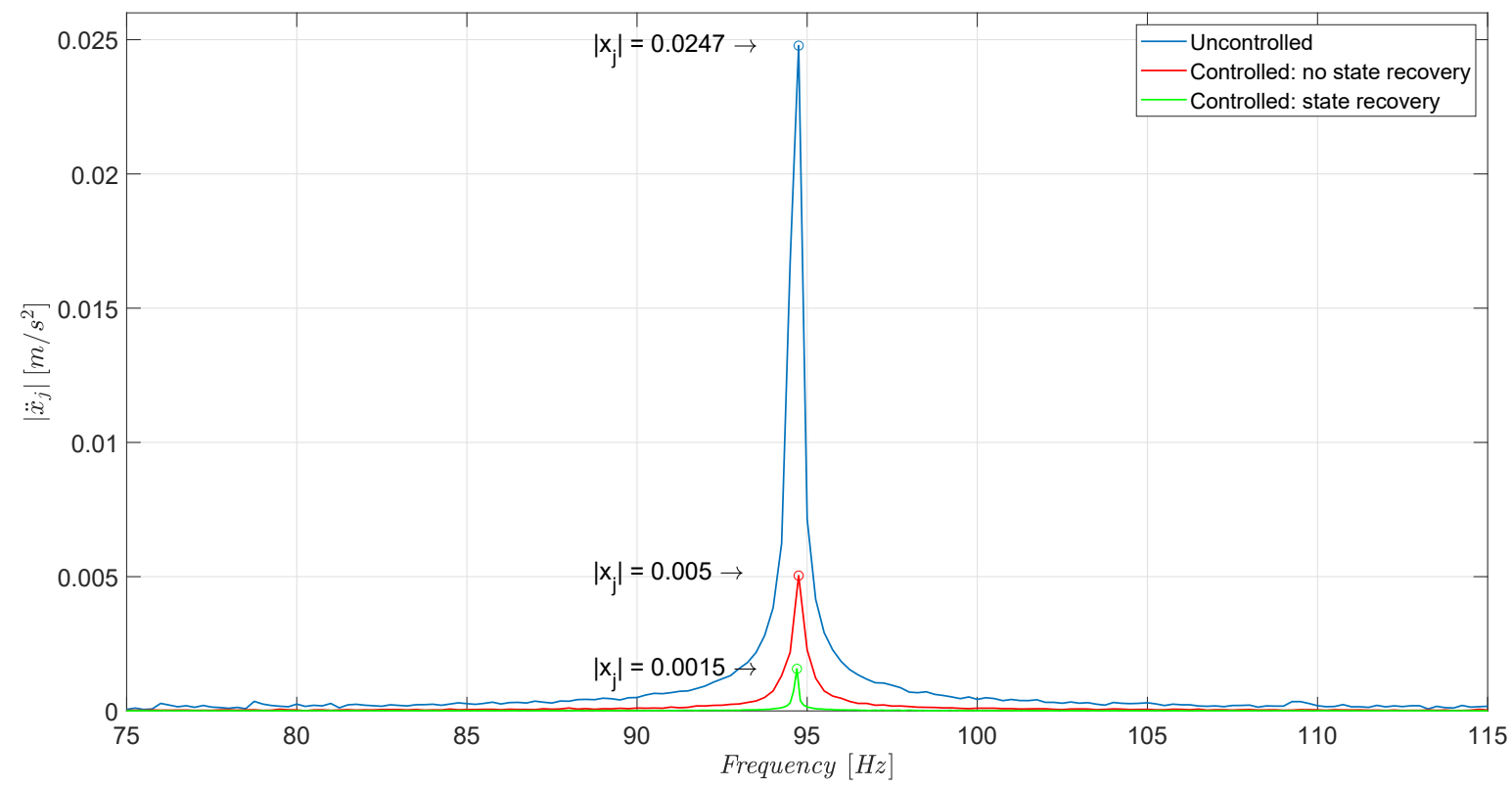

Figure 11: Acceleration signals in the frequency domain in the uncontrolled case (blue line), controlled case without state recovery (red line) and controlled case with state recovery (green line) 
of the acceleration signals depicted in Fig. 10. The uncontrolled case (blue line) is related to the time domain signal up to $5 \mathrm{~s}$ (it is almost the same for both cases); the controlled case is divided between no state recovery (red line, Fig. 10a from $5 \mathrm{~s}$ on) and state recovery (green line, Fig. 10b from $5 \mathrm{~s}$ on).

In the experimental case, compared to the numerical one, the covariance matrix of the error on the measurement $R_{v}$ has been set up in order to obtain an estimate which is very close to the measured value; in fact, a lower error on the measurement has been considered, since the acceleration signal coming from the sensor is previously filtered. The tuning of the value of $R_{v}$ has been carried out by taking one of the time histories of the velocity acquired by the sensor on the board and then by feeding the numerical model with it; at that stage, different values have been tested, until the most suitable one has been found.

\section{CONCLUSIONS}

This work deals with the implementation of a partially decentralized architecture to suppress vibrations, based on the LQG control algorithm. The coupling of the Linear Quadratic Regulator and the Kalman filter requires the knowledge of all the states of the system to be controlled. In this case, each controller can only get one state of the system, which is the one that it can measure with its own sensor, while all the others are unknown. The accuracy of the state estimate provided by the Kalman filter strictly depends on a good knowledge of the system itself and, furthermore, on the availability of a certain number of measured states that will be used by the observer to correct the estimates. Since the real-time sharing of measurements between devices is unfeasible, the so called state recovery algorithm has been investigated in order to limit the use of the wireless communication, still providing enough information to the estimator. Through the numerical analysis it has been demonstrated that such algorithm, triggered by errors on the estimates, and the consequent update of the estimates with the received data, allows to achieve good levels of accuracy of the estimated state. Two different error thresholds have been considered and both of them have been tested on two system configurations, based on a network of two and four stand-alone devices. Experimental tests provide results which are consistent with the numerical ones, although the algorithm is performed with a slightly lower update frequency. However, thanks to a better quality of the estimated state, a more effective control action is guaranteed with respect to the case in which the state recovery is not applied. A reduced model composed by a greater number of vibration modes could be considered and a higher state recovery frequency could be adopted by improving the computational capabilities of the stand-alone device.

\section{REFERENCES}

[1] Lunze, J., [Feedback control of large-scale systems], Prentice Hall (1992).

[2] Elliott, S. J., Gardonio, P., Sors, T. C., and Brennan, M. J., "Active vibroacoustic control with multiple local feedback loops," Acoustical Society of America (2002).

[3] Engels, W. P., Baumann, O. N., and Elliott, S. J., "Centralized and decentralized control of structural vibration and sound radiation," Acoustical Society of America (2005).

[4] Engels, W. P., Baumann, O. N., and Elliott, S. J., "Centralized and decentralized feedback control of kinetic energy," Proceedings of ACTIVE04 (2004).

[5] Balas, M. J., "Feedback control of flexible systems," IEEE Transactions on Automatic Control (1978).

[6] Sun, J. Q., "Some observations on physical duality and colocation of structural control sensors and actuators," Journal of Sound and Vibration (1996).

[7] Inman, D. J., "A comparison of control techniques for large flexible systems," Philosophical Transactions: Mathematical, Physical and Engineering Science (2002).

[8] Lee, C.-K. and Moon, F. C., "Modal sensors/actuators," Journal of Applied Mechanics (1990).

[9] Ploplys, N. J., Kawka, P. A., and Alleyne, A. G., "Closed-loop control over wireless networks," IEEE Control Systems Magazine (2004).

[10] Wang, Y., Lynch, J. P., and Law, K. H., "Decentralized ho controller design for large-scale civil structures," Earthquake Engineering and Structural Dynamics (2008).

[11] Colandairaj, J., Irwin, G. W., and Scanlon, W. G., "Wireless networked control systems with qos-based sampling," IET Control Theory \& Applications (2007). 
[12] Swartz, R. A. and Lynch, J. P., "Strategic network utilization in a wireless structural control system for seismically excited structures," Journal of Structural Engineering (2009).

[13] Chu, S. Y., Soong, T. T., Lin, C. C., and Chen, Y. Z., "Time-delay effect and compensation on direct output feedback controlled mass damper systems," Earthquake Engineering \&3 Structural Dynamics (2002).

[14] Stengel, R. F., [Optimal Control and Estimation] (2012).

[15] Yook, J. K., Tilbury, D. M., and Soparkar, N. R., "Trading computation for bandwidth: reducing communication in distributed control systems using state estimators," IEEE Transactions on Control Systems Technology (2002).

[16] Cinquemani, S. and Braghin, F., "Decentralized active vibration control in cruise ship funnels," Ocean Engineering (2017).

[17] Cinquemani, S., Cazzulani, G., Costa, A., and Resta, F., "Design of a stand-alone active damper for distributed control of vibration," (2016).

[18] Cinquemani, S. and Resta, F., "A mechanical approach to the design of IMSC for vibration suppression," Journal of Vibration and Acoustics (2013).

[19] Cazzulani, G., Resta, F., and Ripamonti, F., "Active modal tuned mass damper for smart structures," Engineering Letters (2011).

[20] Benassi, L., Elliott, S. J., and Gardonio, P., "Active vibration isolation using an inertial actuator with local force feedback control," Journal of Sound and Vibration (2004).

[21] Zimmerman, D. C. and Inman, D. J., "On the nature of the interaction between structures and proof-mass actuators," Journal of Guidance (1989).

[22] Winberg, M., Johansson, S., and Claesson, I., "Inertial mass actuators, understanding and tuning," (2004).

[23] Paulitsch, C., Gardonio, P., Elliott, S. J., Sas, P., and Boonen, R., "Design of a lightweight, electrodynamic, inertial actuator with integrated velocity sensor for active vibration control of a thin lightly-damped panel," Proceedings of the 2004 International Conference on Noise and Vibration Engineering (2004).

[24] Cinquemani, S., Resta, F., and Monguzzi, M., "Limits on the use of inertial actuators in active vibration control," (2007).

[25] Paulitsch, C., Gardonio, P., and Elliott, S. J., "Active vibration damping using an inertial, electrodynamic actuator," Journal of Vibration and Acoustics (2007).

[26] Elliott, S. J., Gardonio, P., Rafaely, B., Harris, R., and Heron, K., "Performance evaluation of a feedback active isolation system with inertial actuators," ISVR Technical Memorandum (1998).

[27] Elliott, S. J., Serrand, M., and Gardonio, P., "Feedback stability limits for active isolation systems with reactive and inertial actuators," Journal of Vibration and Acoustics (2001).

[28] Benassi, L. and Elliott, S. J., "Global control of a vibrating plate using a feedback-controlled inertial actuator," Journal of Sound and Vibration (2005).

[29] Skidmore, G. R. and Hallauer, W. L. J., "Modal-space active damping of a beam-cable structure: theory and experiment," Journal of Sound and Vibration (1985).

[30] Khulief, Y. A., "Vibration suppression in using active modal control," Journal of Sound and Vibration (2001).

[31] Hughes, P. C. and Skelton, R. E., "Controllability and observability of linear matrix-second-order systems," Journal of Applied Mechanics (1980). 